\title{
Efficient myometrial defect closure in a layer by layer fashion after robot-assisted laparoscopic adenomyomectomy: a novel technique
}

\author{
Ayah Hijazi, MD, Youn Jee Chung, MD*, Najeeba Al Sinan, MD, Kyungmin Park, MD, Minji Ko, MD, \\ Jae Yen Song, MD, Mee-Ran Kim, MD, PhD \\ Department of Obstetrics and Gynecology, Seoul St. Mary's Fibroid Center, College of Medicine, The Catholic University of Korea, Seoul, Korea
}

\section{Objective}

In this video, we present our novel technique for myometrial defect closure following robot-assisted laparoscopic adenomyomectomy.

\section{Methods}

A narrated video demonstration of our technique. Our patient was a 47-year-old single woman with severe dysmenorrhea, who did not respond to medical therapy and wished to preserve her uterus. Surgery was performed after thorough counseling and obtaining informed consent from the patient (Institutional Review Board number: KC17OESI0238; approval date: March 19, 2018). After removal of the adenomyotic tissue during surgical intervention, the myometrial defect was closed in three steps. First, the defect between the anterior and posterior innermost myometrial layers was closed using a 2-0 Stratafix suture, CT-1 (circle taper) needle (Ethicon, Somerville, NJ, USA). Next, the two sides were approximated using a 2-0 PDS ${ }^{\circledR}$ (polydioxanone) Suture (Ethicon, Somerville, NJ, USA) and V-34 (TAPERCUT ${ }^{\oplus}$ ) surgical needle (Ethicon, Somerville, NJ, USA). Finally, the serosa was sutured in a baseball fashion using a 2-0 PDS suture, slim half-circle [SH] needle (Ethicon, Somerville, NJ, USA).

\section{Results}

The patient had no postoperative complications, and her pain was greatly improved. The CA125 level decreased from $434 \mathrm{U} / \mathrm{mL}$ to $45.99 \mathrm{U} / \mathrm{mL}$, and the transvaginal ultrasound showed a reduction in posterior myometrial thickness from $5.61 \mathrm{~cm}$ to $2.69 \mathrm{~cm}$.

\section{Conclusion}

This technique maintained the integrity of the endometrial cavity, posterior myometrial thickness, and uterine layer alignment. We believe that it is a feasible technique and may be a solution for adenomyosis in patients seeking for fertility preservation.

Keywords: Adenomyosis; Myometrium; Surgical procedures, Robotic

Received: 2021.01.10. Revised: 2021.02.23. Accepted: 2021.03.11.

Corresponding author: Mee-Ran Kim, MD, PhD

Department of Obstetrics and Gynecology, Seoul St. Mary's Hospital, College of Medicine, The Catholic University of Korea, 222 Banpodaero, Seocho-gu, Seoul 06591, Korea

E-mail: mrkim@catholic.ac.kr

https://orcid.org/0000-0003-4492-0768

*These authors contributed equally to this work. 


\section{Obstetrics \& Gynecology Science}

Ayah Hijazi, et al. Myometrial closure after adenomyomectomy

Adenomyosis, affecting about $20 \%$ of reproductive aged women, is characterized by the presence of endometrial glands within the myometrium, which gets frequently surrounded by hyperplastic and hypertrophic smooth muscle [1]. Adenomyosis mostly involves heavy menstrual bleeding, followed by dysmenorrhea. Less common presentations include dyspareunia and chronic pelvic pain [2]. In recent years, women are more likely to postpone pregnancy towards the end of their reproductive lives; thus, adenomyosis may increasingly become a factor causing infertility, and its diagnosis is an important part of the infertility workup [3].

As more patients are requesting us for fertility preserving management for adenomyosis, we have opted for methods that not only would preserve the uterus but also allow a safe pregnancy. Surgery for adenomyosis had started with the laparotomic approach, followed by less invasive laparoscopy, which was hampered by the loss of tactile sensation, and poorer operative access [4]. Robotic surgery, on the other hand, still allows a minimally invasive procedure, while having the benefit of open surgery with the EndoWrist technology [5].

The defect created in the myometrium following adenomyomectomy can be quite large (Video 1), and maintaining the uterine wall thickness can be a challenge. In our technique, we aimed to maintain normal uterine muscle fiber alignment. This was done by enforcing each layer separately (Fig. 1); we also aimed to keep the endometrium intact, as we believe that its injury might have an effect on future fertility.

Our patient was a 47-year-old woman who was referred to our clinic with severe dysmenorrhea. She had tried medical therapy before with not much improvement. After thorough counseling, we decided to perform robot-assisted laparoscopic adenomyomectomy. In this video, we detail our technique for closure of the myometrial defect using the layerby-layer technique. Maintaining endometrial integrity could
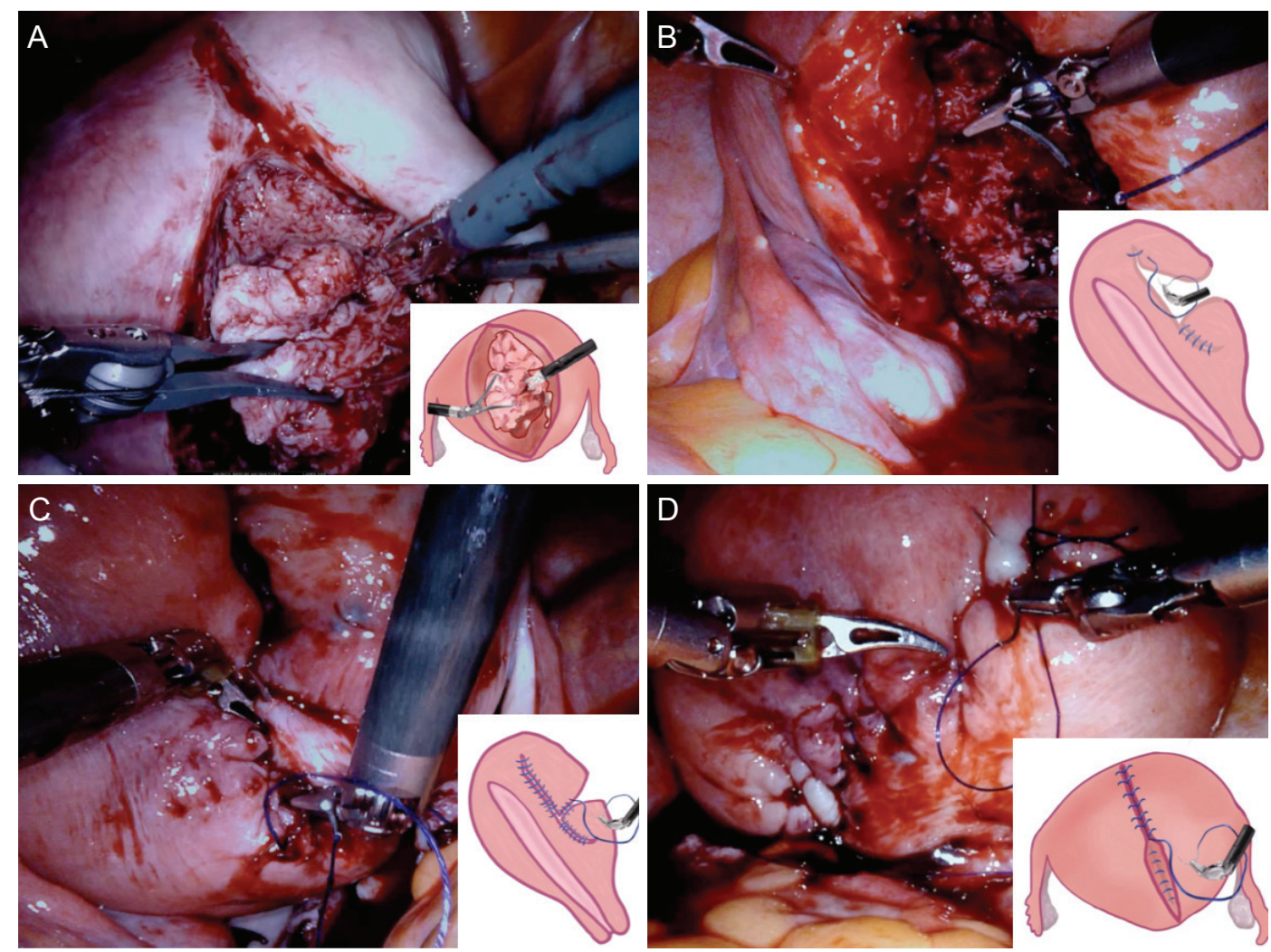

Fig. 1. Surgical procedure. The adenomyotic tissue was grasped using a tenaculum forceps, while dissection was continued using a monopolar curved scissors or a monopolar spatula (A). The defect between the anterior and posterior innermost myometrial layers was closed using a 2-0 stratafix suture, CT-1 needle (B). After the anteroposterior planes were sutured on either side of the incision, the two sides were approximated using a 2-0 PDS suture, V-34 needle (C). Both sides of the serosa were brought together using a 2-0 PDS suture, slim half needle in a baseball fashion (D). 


\section{Obstetrics \& Gynecology Science}

Vol. 64, No. 3, 2021
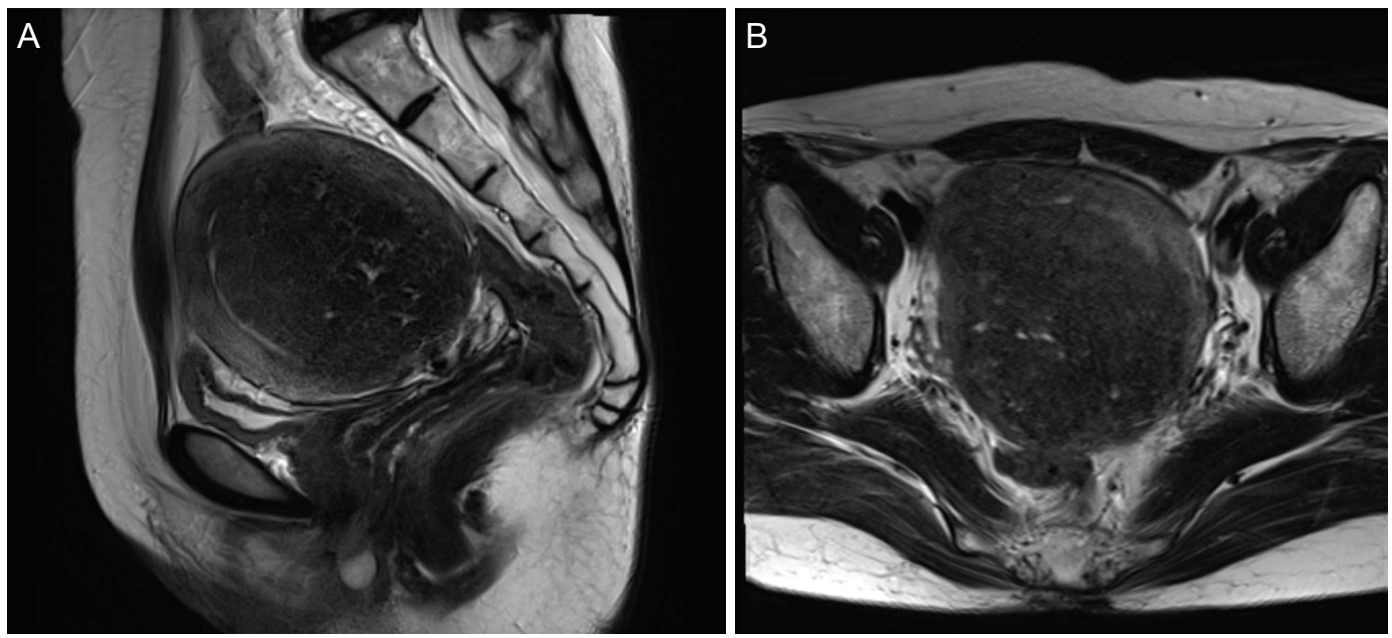

Fig. 2. Pelvic magnetic resonance imaging (MRI). (A) Sagittal and (B) axial pelvic MRI showed adenomyosis involving the posterior uterine wall.
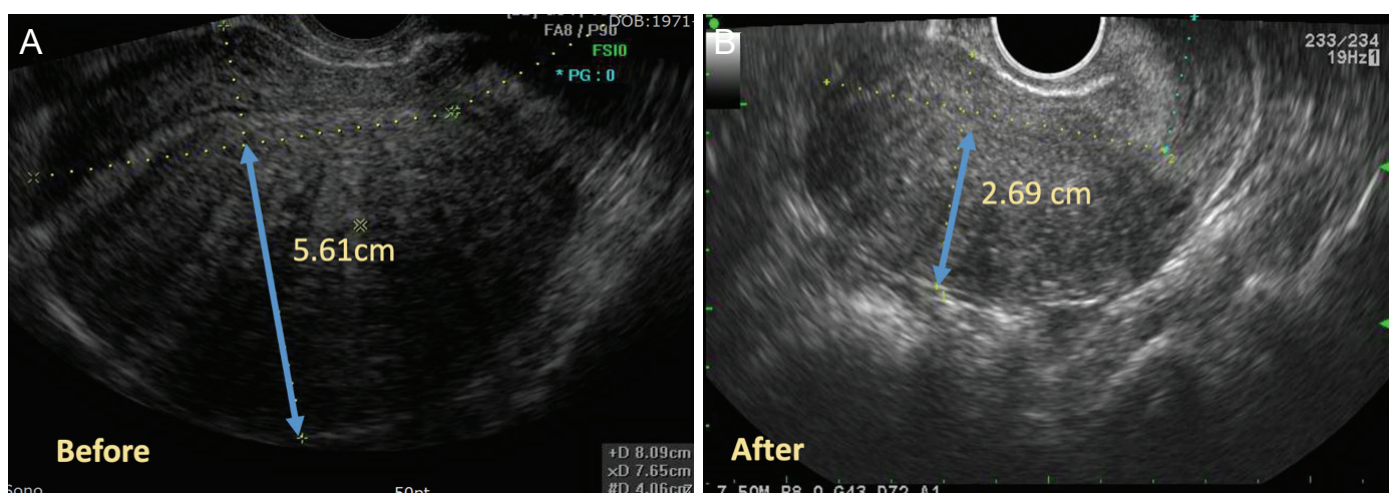

Fig. 3. Transvaginal ultrasound. Follow up transvaginal ultrasound (B) done 2 months postoperatively showed a posterior myometrial thickness of $2.69 \mathrm{~cm}$ compared to the previous measurement of $5.61 \mathrm{~cm}$ in the preoperative ultrasound (A).

be achieved by careful preoperative planning with the aid of pelvic MRI (Fig. 2) and the assistance of intraoperative ultrasound.

The duration of the surgery was approximately 5 hours and 30 minutes, and the estimated blood loss was 500 milliliters. She was discharged on the 3rd postoperative day for social reasons. On follow-up, we found that our patient had minimal dysmenorrhea and was very satisfied. Further, an ultrasound scan, repeated post-surgery, showed a great reduction in the posterior myometrial wall thickness (Fig. 3).

\section{Acknowledgments}

This video has not been published elsewhere, it is not accepted for publication and is not under review for possible publication at any other journal. We presented our video as an oral session in the 48th AAGL Global Congress on MIGS on November 12, 2019.

\section{Conflict of interest}

No potential conflict of interest relevant to this article was reported.

\section{Ethical approval}

This study does not require approval of the Institutional Review Board because no patient data is contained in this article. The study was performed in accordance with the 


\section{Obstetrics \& Gynecology Science}

Ayah Hijazi, et al. Myometrial closure after adenomyomectomy

principles of the Declaration of Helsinki.

\section{Patient consent}

Written informed consent was obtained from the patient for publication of this case report and any accompanying images.

\section{Funding information}

This research was supported by Basic Science Research Program through the National Research Foundation of Korea (NRF) funded by the Ministry of Education (2017R1D1A1B03028045, 2020R1F1A1063199).

\section{Video clip}

Video can be found with this article online at https://doi. org/10.5468/ogs.21025.

\section{References}

1. Donnez J, Donnez O, Dolmans MM. Introduction: uterine adenomyosis, another enigmatic disease of our time. Fertil Steril 2018;109:369-70.

2. Struble J, Reid S, Bedaiwy MA. Adenomyosis: a clinical review of a challenging gynecologic condition. J Minim Invasive Gynecol 2016;23:164-85.

3. Leyendecker G, Kunz G, Kissler S, Wildt L. Adenomyosis and reproduction. Best Pract Res Clin Obstet Gynaecol 2006;20:523-46.

4. Thain S, Tan HH. Approaches to adenomyomectomy. Gynecol Minim Invasive Ther 2015;4:49-54.

5. Sinha R, Sanjay M, Rupa B, Kumari S. Robotic surgery in gynecology. J Minim Access Surg 2015;11:50-9. 